\title{
Thrombectomy within 8 Hours after Symptom Onset in Ischemic Stroke
}

\author{
T.G. Jovin, A. Chamorro, E. Cobo, M.A. de Miquel, C.A. Molina, A. Rovira, \\ L. San Román, J. Serena, S. Abilleira, M. Ribó, M. Millán, X. Urra, P. Cardona, \\ E. López-Cancio, A. Tomasello, C. Castaño, J. Blasco, L. Aja, L. Dorado, \\ H. Quesada, M. Rubiera, M. Hernández-Pérez, M. Goyal, A.M. Demchuk, \\ R. von Kummer, M. Gallofré, and A. Dávalos, for the REVASCAT Trial Investigators*
}

ABSTRACT

The authors' full names, academic degrees, and affiliations are listed in the Appendix. Address reprint requests to Dr. Dávalos at the Department of Neurosciences, Hospital Germans Trias I Pujol, Universitat Autònoma de Barcelona, Avda Canyet, Badalona 08916, Barcelona, Spain, or at adavalos.germanstrias@gencat.cat.

Drs. Jovin and Dávalos contributed equally to this article.

*A complete list of sites and investigators in the Randomized Trial of Revascularization with Solitaire FR Device versus Best Medical Therapy in the Treatmen of Acute Stroke Due to Anterior Circulation Large Vessel Occlusion Presenting within Eight Hours of Symptom Onset (REVASCAT) is provided in the Supplementary Appendix, available at NEJM.org.

This article was published on April 17, 2015, at NEJM.org.

N Engl J Med 2015;372:2296-306. DOI: 10.1056/NEJMoa1503780

Copyright ( 2015 Massachusetts Medical Society.

\section{BACKGROUND}

We aimed to assess the safety and efficacy of thrombectomy for the treatment of stroke in a trial embedded within a population-based stroke reperfusion registry.

\section{METHODS}

During a 2-year period at four centers in Catalonia, Spain, we randomly assigned 206 patients who could be treated within 8 hours after the onset of symptoms of acute ischemic stroke to receive either medical therapy (including intravenous alteplase when eligible) and endovascular therapy with the Solitaire stent retriever (thrombectomy group) or medical therapy alone (control group). All patients had confirmed proximal anterior circulation occlusion and the absence of a large infarct on neuroimaging. In all study patients, the use of alteplase either did not achieve revascularization or was contraindicated. The primary outcome was the severity of global disability at 90 days, as measured on the modified Rankin scale (ranging from 0 [no symptoms] to 6 [death]). Although the maximum planned sample size was 690 , enrollment was halted early because of loss of equipoise after positive results for thrombectomy were reported from other similar trials.

\section{RESULTS}

Thrombectomy reduced the severity of disability over the range of the modified Rankin scale (adjusted odds ratio for improvement of 1 point, 1.7; 95\% confidence interval [CI], 1.05 to 2.8) and led to higher rates of functional independence (a score of 0 to 2 ) at 90 days ( $43.7 \%$ vs. $28.2 \%$; adjusted odds ratio, 2.1; $95 \%$ CI, 1.1 to 4.0 ). At 90 days, the rates of symptomatic intracranial hemorrhage were $1.9 \%$ in both the thrombectomy group and the control group $(\mathrm{P}=1.00)$, and rates of death were $18.4 \%$ and $15.5 \%$, respectively $(\mathrm{P}=0.60)$. Registry data indicated that only eight patients who met the eligibility criteria were treated outside the trial at participating hospitals.

\section{CONCLUSIONS}

Among patients with anterior circulation stroke who could be treated within 8 hours after symptom onset, stent retriever thrombectomy reduced the severity of poststroke disability and increased the rate of functional independence. (Funded by Fundació Ictus Malaltia Vascular through an unrestricted grant from Covidien and others; REVASCAT ClinicalTrials.gov number, NCT01692379.) 
ECENTLY COMPLETED PROSPECTIVE, randomized trials involving patients with acute stroke have consistently shown a clinical benefit for mechanical thrombectomy. ${ }^{1-4}$ Our study, called the Randomized Trial of Revascularization with Solitaire FR Device versus Best Medical Therapy in the Treatment of Acute Stroke Due to Anterior Circulation Large Vessel Occlusion Presenting within Eight Hours of Symptom Onset (REVASCAT), shares the following four features with the previously cited trials: enrollment limited to patients with imaging-based evidence of proximal occlusion of the M1 segment (main trunk) of the middle cerebral artery with or without concomitant occlusion of the internal carotid artery, imaging-based exclusion of patients with a large core (indicating large cerebral infarct), use of a stent retriever, and ongoing quality-improvement efforts to reduce the time to reperfusion to the minimum.

The lack of consecutive enrollment has been identified as a major shortcoming in endovascular stroke trials. ${ }^{5}$ To account for this potential limitation by determining the extent of consecutive enrollment, the study was conducted within Sistema Online d'Informació de l'Ictus Agut de Catalunya (SONIIA), a concomitant, populationbased registry of acute stroke reperfusion procedures $^{6}$ that captured patients within the same catchment area as that of the participating hospitals in our study, including patients who were treated in the thrombectomy group.

The aim of this study was to determine the efficacy and safety of neurovascular thrombectomy with the Solitaire stent retriever in conjunction with medical therapy versus medical therapy alone, among eligible patients with acute ischemic stroke that could be treated within 8 hours after the onset of symptoms and to assess the proportion of eligible patients who were treated outside the study.

\section{METHODS}

\section{STUDY DESIGN AND OVERSIGHT}

REVASCAT was a multicenter, prospective, randomized, sequential, open-label phase 3 study with blinded evaluation. We compared thrombectomy with medical therapy alone in eligible patients who had received intravenous alteplase within 4.5 hours after the onset of symptoms without revascularization after 30 minutes of al- teplase infusion or who had a contraindication to intravenous alteplase. The trial was monitored by an independent data and safety monitoring board.

The steering committee designed the study, had unrestricted access to the data, and together with all other coauthors reviewed the analysis with study statisticians, wrote the first and subsequent drafts of the manuscript, and attest to the integrity of the trial, the fidelity of this report to the study protocol, and the completeness and accuracy of the reported data. The study was funded by an unrestricted grant from the manufacturer of the stent retriever (Covidien), which was not involved in the design or conduct of the study or in the writing of the protocol or the manuscript.

The study protocol, ${ }^{7}$ which is available with the full text of this article at NEJM.org, was approved by a central medical ethics committee and by the research board at each participating center. All patients or their surrogates provided written informed consent.

\section{PATIENTS}

From November 2012 through December 2014, we screened patients at four study centers in Catalonia, Spain. Eligible patients were between the ages of 18 and 80 years, had an occlusion in the proximal anterior circulation that could be treated within 8 hours after symptom onset, had a prestroke functional ability of 1 or less on the modified Rankin scale (ranging from 0 [no symptoms] to 6 [death]), and had a baseline score of at least 6 points on the National Institutes of Health Stroke Scale (NIHSS), which ranges from 0 to 42, with higher values indicating more severe deficit. The main exclusion criteria on imaging were evidence of a large ischemic core, as indicated by an Alberta Stroke Program Early Computed Tomography Score (ASPECTS) of less than 7 on computed tomography (CT) without the use of contrast material or a score of less than 6 on diffusion-weighted magnetic resonance imaging (MRI). (ASPECTS values range from 0 to 10 , with higher values indicating less infarct burden.) Additional details with respect to imaging requirements, interventions, and clinical and radiologic assessments are provided in the Supplementary Appendix, available at NEJM.org.

After the enrollment of 160 patients, the inclusion criteria were modified to include patients up to the age of 85 years with an ASPECTS 
score of more than 8. Detailed clinical and imaging criteria for inclusion and exclusion are provided in Table S1 in the Supplementary Appendix.

\section{STUDY SITES AND INTERVENTIONISTS}

Study sites consisted of certified comprehensive stroke centers that treat more than 500 patients with acute stroke and perform more than 60 mechanical stroke thrombectomy procedures annually and are staffed by trained neurointerventionalists who are required to have performed at least 20 thrombectomies with the Solitaire device. Participating sites were the four large, designated, comprehensive stroke centers in Catalonia, Spain, covering a defined population of 7.5 million. The Catalan Health Authorities monitored adherence to the medical-therapy guidelines at the sites (see the Methods section in the Supplementary Appendix).

\section{COMPREHENSIVE REGISTRY}

During the trial, all reperfusion treatments (endovascular or intravenous) for acute stroke in Catalonia were captured through SONIIA, a prospective, population-based, and externally monitored registry regulated by the Catalan Department of Health. (Details regarding the SONIIA registry are provided in the Supplementary Appendix.)

\section{RANDOMIZATION}

We randomly assigned 206 patients in a 1:1 ratio to receive either medical therapy (including intravenous alteplase when eligible) and endovascular treatment with the Solitaire stent retriever (thrombectomy group) or medical therapy alone (control group). We used a real-time computerized randomization procedure that was stratified according to age ( $\leq 70$ or $>70$ years), baseline NIHSS score ( 6 to 16 or $\geq 17$ ), therapeutic window $(\leq 4.5$ or $>4.5$ hours), occlusion site (intracranial internal carotid artery or M1 segment [main trunk of the middle cerebral artery]), and participating center.

\section{PRIMARY OUTCOME}

The primary outcome was the severity of disability at 90 days, according to the distribution of scores on the modified Rankin scale. ${ }^{8}$ Severe disability (score of 5) and death (score of 6) were combined into a single, worst category. ${ }^{1,2,9}$ Local and external certified assessors who were unaware of study-group assignments separately evaluated the primary outcome variable in each patient by means of a structured interview. ${ }^{10}$ The primary analysis was based on central evaluation by means of video recording (in 106 evaluations). In cases in which the video recording was unavailable, outcomes as determined in person by local investigators in a blinded manner were used as default (in 65 evaluations). Sensitivity analyses were performed with outcome determinations in a blinded manner by local investigators and central readers. Decisions regarding the adjudication method for the primary outcome were made by the steering committee in a blinded manner before the first interim analysis. (Additional details are provided in the Methods section in the Supplementary Appendix.)

\section{SECONDARY AND SAFETY OUTCOMES}

Secondary outcomes included centrally adjudicated infarct volumes on CT or MRI at 24 hours, vessel revascularization on CT angiography (CTA) or MR angiography (MRA) at 24 hours (data not yet analyzed), early dramatic response to treatment (defined as a decrease in the NIHSS score of $\geq 8$ from baseline or an NIHSS score of 0 to 2 at 24 hours), the NIHSS score and Barthel Index (with the latter on a scale from 0 to 100 , with higher scores indicating less disability) at 90 days, and health status, as measured on the EuroQol Group 5-Dimension Self-Report Questionnaire (EQ-5D, on a scale of -0.33 to 1 , with higher values indicating a better quality of life) at 90 days. In the thrombectomy group, the core laboratory used post-procedure conventional angiography to adjudicate successful vessel revascularization, which was defined as a grade of $2 b$ or 3 (indicating reperfusion of more than $50 \%$ of the affected territory) on the modified Thrombolysis in Cerebral Infarction (mTICI) scale of 0 to 3, with higher grades indicating increased reperfusion.

Safety outcomes were the rates of death and symptomatic intracranial hemorrhage at 90 days, as confirmed on neuroimaging (CT or MRI) evaluated by the core laboratory, primarily according to the definition used in the Safe Implementation of Thrombolysis in Stroke-Monitoring Study (SITS-MOST) ${ }^{11}$ which was parenchymal hemorrhage type 2 on follow-up imaging and neurologic deterioration of at least 4 points on the NIHSS. Evaluators also used the definition of symptomatic intracranial hemorrhage that was used in the second European-Australasian Acute 
Stroke Study (ECASS II) ${ }^{12}$ - namely, any type of intracerebral hemorrhage on post-treatment imaging with an increase of at least 4 points on the NIHSS. An independent clinical events committee adjudicated safety outcomes, procedure-related complications (e.g., arterial perforation, arterial dissection, and embolization in a previously uninvolved vascular territory), and serious adverse events.

\section{STATISTICAL ANALYSIS}

All analyses were performed in the intention-totreat population. The measure of effect size was a cumulative odds ratio as calculated by logistic regression (shift analysis). This analysis relies on the assumption of an odds ratio behind any cutoff point, which has been shown to be robust to minor deviations. ${ }^{8}$ The primary analysis has been adjusted both for minimization factors and for the intravenous use of alteplase. We calculated that the enrollment of 690 patients would provide a power of $90 \%$ to detect a difference in the distribution of scores on the modified Rankin scale with a one-sided significance level of 0.025 in the analysis of the primary outcome, assuming an expected result of an odds ratio of 1.615. Because of the uncertainty about the size of the treatment effect for the primary outcome, REVASCAT was designed as a sequential study. On the basis of stopping boundaries for the Whitehead triangular test, we planned to conduct a maximum of four equally spaced reviews when enrollment had reached approximately $25 \%, 50 \%, 75 \%$, and $100 \%$ of the sample size. We assigned lower individual limits of significance to the four analyses in order to achieve an overall shared one-sided alpha level of 0.025 .

\section{TRIAL TERMINATION}

The first interim analysis was performed as planned after $25 \%$ patients ( 174 of the maximum sample size) had completed 90 days of follow-up. The steering committee accepted the recommendation of the data and safety monitoring board to stop recruitment because of loss of equipoise (as described in detail in the Supplementary Appendix). Although the interim results did not reach the prespecified stopping boundaries (see the results of the first interim analysis in Fig. S6-1 in the Supplementary Appendix), study recruitment was terminated because of emerging results from three other studies ${ }^{2-4}$ that showed the efficacy of thrombectomy, which raised ethical concerns about further assignment of patients to the control group. Since the trial was stopped because the primary hypothesis was no longer an open question, we changed our goal from hypothesis testing to estimation. Since just one analysis was performed, adjustment for multiple comparisons was no longer required, and $95 \%$ confidence intervals are reported (details are provided in the Supplementary Appendix). All reported 95\% confidence intervals and $\mathrm{P}$ values are nominal and based on the recorded data.

RESULTS

\section{PATIENTS}

A total of 206 patients were enrolled in the study (Fig. S1 in the Supplementary Appendix). One patient withdrew consent immediately after randomization and was not included in the analysis. No crossovers occurred. All patients had an available evaluation at 90 days for the primary outcome. In 5 patients with a score of 4 or 5 on the modified Rankin scale, the video evaluation was replaced by a telephone interview. The intention-to-treat analysis included 103 patients in the thrombectomy group and 103 in the control group.

During the study period, 2576 patients with ischemic stroke were included in the SONIIA registry, making up $15.6 \%$ of all admissions for ischemic stroke in Catalonia and resulting in an aggregate reperfusion-therapy rate of 17 per 100,000 residents of Catalonia per year. Intravenous thrombolysis alone was used in $2036 \mathrm{pa}-$ tients (79\%). The remaining 540 patients $(21 \%)$ underwent endovascular therapy, including 260 who underwent a combination of intravenous alteplase and thrombectomy (Fig. S2 and S3 in the Supplementary Appendix). Of the 540 patients who underwent thrombectomy, 464 (86\%) were treated at hospitals included in our study.

Periodic review of registry data and evaluation of medical records at local sites that was intended to confirm eligibility revealed that 111 of 464 patients receiving endovascular therapy met the study criteria; of these patients, 103 were treated in our study. Therefore, only 8 patients who were eligible to participate in our study received endovascular therapy outside the trial at participating hospitals (Table S3 and Fig. S3 in the Supplementary Appendix). 


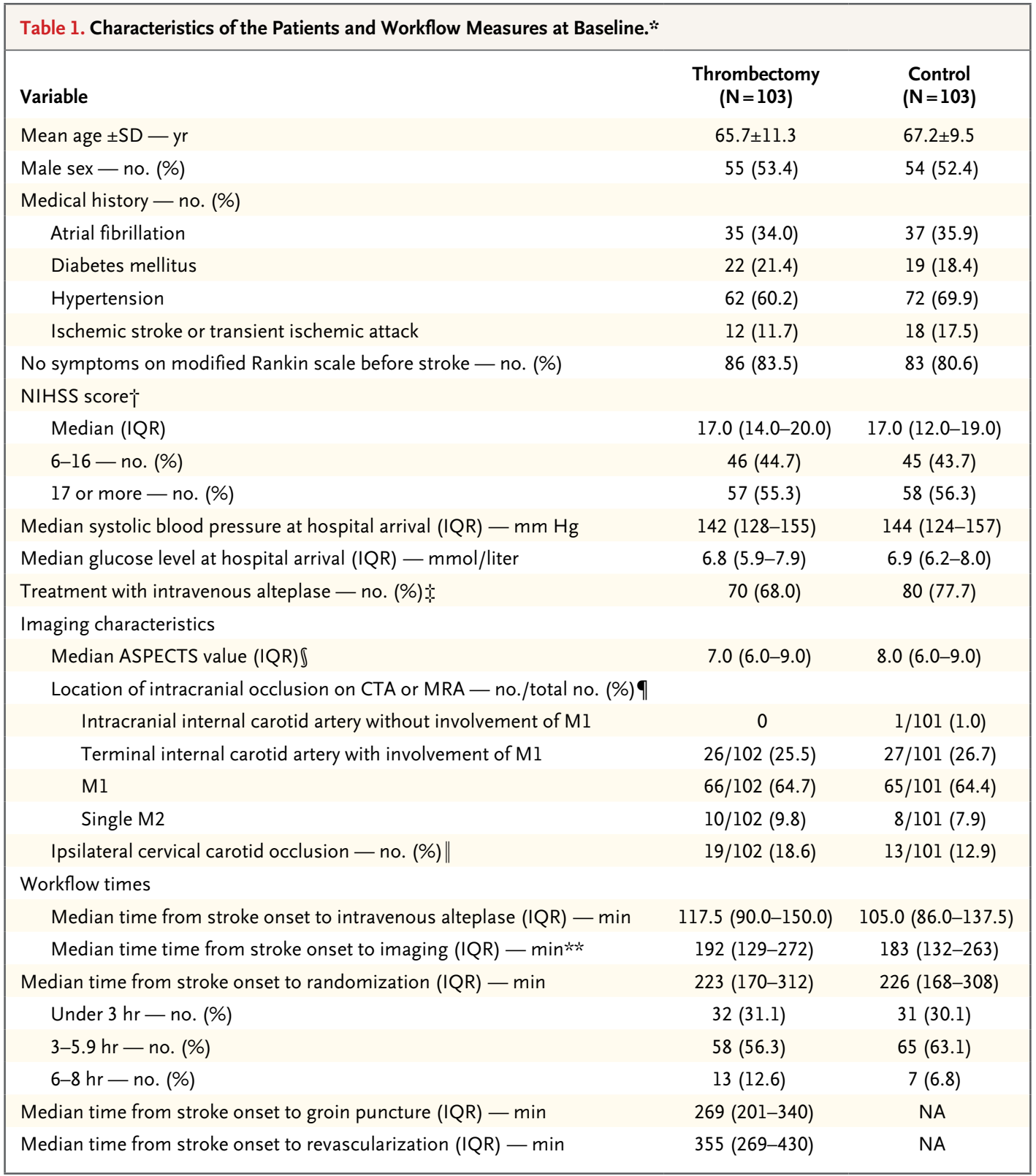

* There were no significant differences between the two groups. To convert the values for glucose to milligrams per deciliter, divide by 0.05551 . CTA denotes computed tomography angiography, IQR interquartile range, Ml main trunk of the middle cerebral artery, M2 first-order branch of the main trunk of the middle cerebral artery, MRA magnetic resonance angiography, and NA not applicable.

$\dagger$ Scores on the National Institutes of Health Stroke Scale (NIHSS) range from 0 to 42, with higher scores indicating more severe neurologic deficits.

$\ddagger$ Intravenous alteplase was administered before randomization, except in one patient, and at least 30 minutes before confirmation of the target artery occlusion on CTA or MRA. Contraindications for intravenous alteplase according to group are provided in Table S4 in the Supplementary Appendix.

$\int$ The Alberta Stroke Program Early Computed Tomography Score (ASPECTS) is an imaging measure of the extent of ischemic stroke. Scores range from 0 to 10 , with higher scores indicating a smaller infarct core. Listed are values for the core laboratory assessment.

9. The location of the occlusion was not available in 1 patient in the intervention group and 2 patients in the control group.

| Ipsilateral carotid stenting was performed in nine patients $(8.7 \%)$ in the thrombectomy group.

*** The listed time corresponds to the imaging performed before randomization at least 30 minutes after the initiation of intravenous alteplase (in patients in whom the drug was administered). 


\begin{tabular}{|c|c|c|c|c|c|}
\hline Outcome & $\begin{array}{l}\text { Thrombectomy } \\
\qquad(\mathrm{N}=103)\end{array}$ & $\begin{array}{l}\text { Control } \\
(N=103)\end{array}$ & $\begin{array}{c}\text { Effect } \\
\text { Variable }\end{array}$ & $\begin{array}{l}\text { Unadjusted } \\
\text { Value } \\
(95 \% \mathrm{Cl})\end{array}$ & $\begin{array}{l}\text { Adjusted } \\
\text { Value } \\
(95 \% \mathrm{CI})\end{array}$ \\
\hline $\begin{array}{l}\text { Primary outcome: score on modified Rankin } \\
\text { scale at } 90 \text { days }\end{array}$ & NA & NA & $\begin{array}{l}\text { Common } \\
\text { odds ratio }\end{array}$ & 1.7 (1.04 to 2.7$)$ & 1.7 (1.05 to 2.8$)$ \\
\hline \multicolumn{6}{|l|}{ Secondary outcome } \\
\hline $\begin{array}{l}\text { Score of } 0 \text { to } 2 \text { on modified Rankin scale } \\
\text { at } 90 \text { days - no. }(\%) \dagger\end{array}$ & $45(43.7)$ & $29(28.2)$ & Odds ratio & 2.0 (1.1 to 3.5$)$ & 2.1 (1.1 to 4.0$)$ \\
\hline $\begin{array}{l}\text { Dramatic neurologic improvement at } 24 \mathrm{~h} \\
\quad \text { - no./total no. (\%) }\end{array}$ & $59 / 100(59.0)$ & $20 / 100(20.0)$ & Odds ratio & $5.5(2.9$ to 10.3$)$ & $5.8(3.0$ to 11.1$)$ \\
\hline Median NIHSS score at 90 days (IQR) & $2.0(0.0$ to 8.0$)$ & $6.0(2.0$ to 11.0$)$ & Beta & $-2.7(-4.4$ to -0.9$)$ & $-2.4(-4.1$ to -0.8$)$ \\
\hline $\begin{array}{l}\text { Barthel Index score of } 95 \text { to } 100 \text { at } 90 \text { days } \\
\qquad \text { no./total no. (\%) } \mathbb{}\end{array}$ & $47 / 82(57.3)$ & $23 / 87(26.4)$ & Odds ratio & 3.7 (2.0 to 7.1$)$ & $4.2(2.1$ to 8.4$)$ \\
\hline Median EQ-5D score at 90 days (IQR) & $0.65(0.21$ to 0.79$)$ & 0.32 (0.13 to 0.70$)$ & Beta & 0.13 (0.03 to 0.23$)$ & 0.11 (0.02 to 0.21$)$ \\
\hline \multicolumn{6}{|l|}{ Infarct volume at $24 \mathrm{hr}$} \\
\hline $\begin{array}{l}\text { Patients evaluated on CT:diffusion- } \\
\text { weighted } \mathrm{MRI} \text { — no. }\end{array}$ & $91: 10$ & $98: 5$ & & & \\
\hline Median (IQR) - ml & $16.3(8.3$ to 58.5$)$ & 38.6 (11.9 to 86.8$)$ & $P$ value $\|$ & $P=0.02$ & \\
\hline \multicolumn{6}{|c|}{$\begin{array}{l}\text { CT denotes computed tomography, and MRI magnetic resonance imaging. } \\
\text { The modified Rankin scale of functional disability ranges from } 0 \text { (no symptoms) to } 6 \text { (death). } \\
\text { Scores at } 24 \text { hours were not available for } 3 \text { patients in each group because of early death or intubation. } \\
\text { Scores on the Barthel Index range from } 0 \text { to } 100 \text {, with higher values indicating good performance of daily living activities. A score between } \\
95 \text { and } 100 \text { indicates no disability that interferes with daily activities. Included are patients who were alive at } 90 \text { days. } \\
9 \text { Scores on the EuroQoL Group 5-Dimension Self-Report Questionnaire (EQ-5D) range from - } 0.33 \text { to } 1 \text {, with higher scores indicating a better } \\
\text { quality of life. }\end{array}$} \\
\hline
\end{tabular}

\section{BASELINE CHARACTERISTICS}

Baseline characteristics were similar in the two study groups (Table 1, and Table S4 in the Supplementary Appendix). The median NIHSS score was 17, the median ASPECTS score was 7 in the thrombectomy group and 8 in the control group, and intravenous alteplase was administered to $68.0 \%$ of patients in the thrombectomy group and $77.7 \%$ of those in the control group. The median time from stroke onset to randomization was 225 minutes.

\section{INTERVENTION DETAILS}

Thrombectomy was performed in 98 of 103 patients in the thrombectomy group. Ipsilateral carotid stenting was performed in 9 patients. Seven procedures in the thrombectomy group (6.7\%) were performed under general anesthesia. Outside of protocol, 1 patient was treated with intracranial angioplasty after failed attempts with the stent retriever, and 1 patient received intraarterial alteplase. Workflow metrics and procedural char- acteristics are provided in Table 1, and Table S5 in the Supplementary Appendix.

\section{PRIMARY OUTCOME}

The primary outcome analysis showed a common odds ratio of improvement in the distribution of the modified Rankin scale score of 1.7 (95\% confidence interval [CI], 1.05 to 2.8) favoring thrombectomy (Table 2 and Fig. 1). The absolute betweengroup difference in the proportion of patients who were functionally independent (score of 0 to 2 on the modified Rankin scale) was 15.5 percentage points, favoring thrombectomy ( $43.7 \%$ vs. $28.2 \%$; adjusted odds ratio, 2.1; 95\% CI, 1.1 to 4.0 ) (Table 2).

\section{SECONDARY OUTCOMES}

Secondary outcomes also favored the thrombectomy group (Table 2). Successful revascularization was achieved in $66 \%$ of patients in the thrombectomy group according to core laboratory assessments (Table 3) and in $80 \%$ of the patients according to the assessments of local 


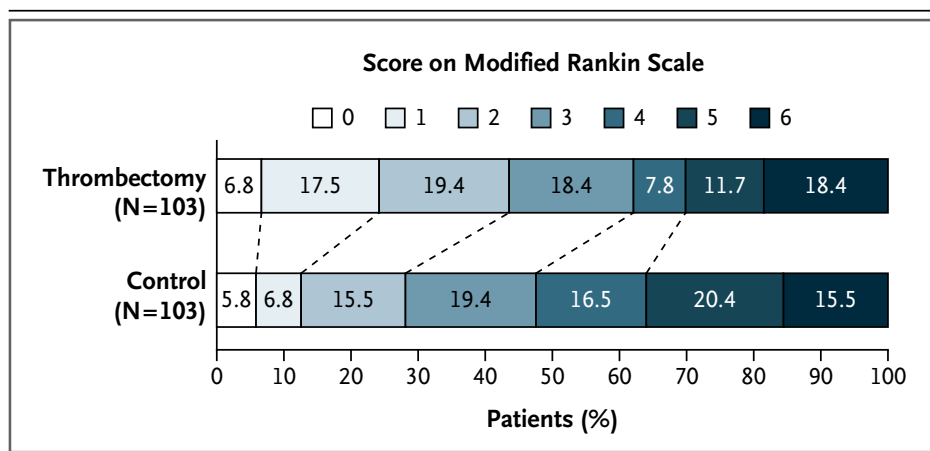

Figure 1. Distribution of Functional Scores at 90 Days (Intention-to-Treat Population).

Shown are scores on the modified Rankin scale for patients in the thrombectomy group and the control group who were evaluated by means of video recording (in 106 patients) and by local investigators (in 65 patients). Scores on the modified Rankin scale range from 0 to 6 , with 0 indicating no symptoms; 1 , no clinically significant disability; 2 , slight disability (able to handle own affairs without assistance but unable to carry out all previous activities); 3 , moderate disability requiring some help, but able to walk unassisted; 4 , moderately severe disability (unable to attend body needs and unable to walk); 5 , severe disability (requiring constant nursing care and attention); and 6 , death. Scores of 5 and 6 were combined for the analysis. A significant difference between the thrombectomy group and the control group was noted in the overall distribution of scores (adjusted common odds ratio for improvement of 1 point on the modified Rankin scale, $1.7 ; 95 \%$ confidence interval, 1.05 to 2.8 ).

interventionalists. (For details, see Table S5 in the Supplementary Appendix.)

\section{SAFETY VARIABLES}

There were no significant between-group differences between the thrombectomy group and the control group in the rates of death $(18.4 \%$ vs. $15.5 \%, \mathrm{P}=0.60$ ) or symptomatic intracranial hemorrhage $(1.9 \%$ in each group, $\mathrm{P}=1.00)$ at 90 days. Rates of other serious adverse events during the 90-day follow-up period were also similar in the two study groups (Table 4). A complete list of adverse events is provided in Tables S6 and S7 in the Supplementary Appendix.

\section{SUBGROUP ANALYSES}

We did not detect significant differences in the effect of thrombectomy in prespecified subgroup analyses. However, the relatively small sample size resulting from the early termination of the trial reduced the power of these planned analyses (Fig. 2, and Fig. S5 in the Supplementary Appendix).

\section{SENSITIVITY ANALYSES}

The primary outcome analysis that was based only on the local evaluator's adjudication in a blinded manner showed higher treatment effects for thrombectomy (odds ratio, 1.9; 95\% CI, 1.1 to 3.2) than the above-mentioned odds ratios. Details regarding the sensitivity analyses are provided in Figure S6 in the Supplementary Appendix.

DISCUSSION

Our study findings are consistent with those of recently reported trials ${ }^{1-4}$ showing that in patients with acute stroke caused by a proximal large-vessel occlusion and an absence of a large infarct on baseline imaging, mechanical thrombectomy with the Solitaire stent retriever was safe and led to improved clinical outcomes, as compared with medical therapy alone. Rates of functional independence were increased by 15.5 percentage points in the thrombectomy group, which means that 6.5 patients would need to be treated with thrombectomy to prevent one case of functional dependency or death. Thrombectomy was associated with a shift toward better outcomes across the entire spectrum of disability.

There are important features that distinguish our study from other similar trials. ${ }^{1-4,13}$ Our study was embedded into a concomitant reperfusion registry that captured all endovascular stroke treatments that were performed in the target population during the study. As such, we had access to data regarding the number of potentially eligible patients who were treated outside the study at participating centers and over the entire target population. The vast majority of patients who were treated with endovascular therapy and who met the eligibility criteria for our study were treated within our study.

We aimed to avoid inclusion of patients who had an early response to intravenous alteplase. A prerequisite for enrollment was imaging proof of the presence of a qualifying occlusion $30 \mathrm{~min}-$ utes after the administration of alteplase, a factor that partially explains the longer times from hospital arrival to reperfusion and the lower rates of mTICI scores of $2 b$ or 3 in our study, as compared with values in the Solitaire with the Intention for Thrombectomy as Primary Endovascular Treatment (SWIFT PRIME) study, ${ }^{1}$ the Endovascular Treatment for Small Core and Anterior Circulation Proximal Occlusion with Emphasis on Minimizing CT to Recanalization Times (ESCAPE) study, ${ }^{2}$ and the Extending the Time for Thrombolysis in Emergency Neurological Deficits-Intra-arterial (EXTEND-IA) study. ${ }^{3}$ 


\begin{tabular}{|c|c|c|}
\hline \multicolumn{3}{|l|}{ Group.* } \\
\hline \multirow[t]{2}{*}{ Score } & At Baseline & After Treatment \\
\hline & \multicolumn{2}{|c|}{ no. of patients (\%) } \\
\hline 0 : No reperfusion & $84(82.4)$ & $8(7.8)$ \\
\hline $\begin{array}{l}\text { 1: Antegrade flow past the initial occlusion but limited distal branch filling } \\
\text { with little or slow distal reperfusion }\end{array}$ & $10(9.8)$ & $2(2.0)$ \\
\hline 2a: Antegrade reperfusion of less than $50 \%$ of the previously ischemic territory & $4(3.9)$ & $25(24.5)$ \\
\hline $\begin{array}{l}\text { 2b: Antegrade reperfusion of more than } 50 \% \text { of the previously ischemic } \\
\text { territory }\end{array}$ & $1(1.0)$ & $48(47.1)$ \\
\hline $\begin{array}{l}\text { 3: Complete antegrade reperfusion of the previously ischemic territory, with } \\
\text { absence of visualized occlusion in all distal branches }\end{array}$ & $2(2.0)$ & $19(18.6)$ \\
\hline Could not be assessed & $1(1.0)$ & 0 \\
\hline
\end{tabular}

$* \mathrm{P}<0.001$ for the overall comparison between baseline values and values after treatment by McNemar's test for trend. Listed are data obtained on angiography during the core laboratory evaluation. Data were missing for one patient.

Given the rigorous vetting process for interventionists that was applied in our study, it is unlikely that the observed differences in reperfusion rates were due to potential differences in operator skill. It is more likely that these differences were due to the fact that the time from symptom onset to randomization was longer in our study and inclusion criteria resulted in a study population enriched with patients in whom occlusive lesions had a reduced response to thrombectomy attempts, similar to the patients enrolled in the Multicenter Randomized Clinical Trial of Endovascular Treatment for Acute Ischemic Stroke in the Netherlands (MR CLEAN). ${ }^{4}$

In our study, imaging that was used for the selection of patients was performed in the majority of cases with widely available methods (CT and CTA) and aimed to confirm large-vessel occlusion while excluding patients with largeischemic core. Similar to findings in other studies, we observed discrepancies between central and investigator-adjudicated ASPECTS scores and occlusion sites. ${ }^{1,2}$ Centrally adjudicated imaging studies showed that $25 \%$ of the patients had ASPECTS scores of 6 or less and $9 \%$ had occlusions in the M2 segment (first-order branch of the main trunk of the middle cerebral artery), which constituted an exclusion criteria. If enrollment had been carried out on the basis of centrally adjudicated requirements for imaging, a significant proportion of patients who were enrolled in our study might have been excluded, which highlights some of the challenges associated with conducting imaging-based endovascular stroke trials. It can be argued, however, that inclusion according to investigator-based interpretation of imaging is a more accurate reflection of real-world clinical practice.

Because ASPECTS interpretation is less accurate in estimating the core volume than are other imaging methods, ${ }^{14}$ our imaging-selection requirements may have allowed the inclusion of patients with infarct sizes that were larger than those in patients included in other trials..$^{1-3}$ This factor, in conjunction with longer times from symptom onset to reperfusion, centrally adjudicated outcomes, and imbalances in the proportion of patients with extracranial occlusion of the internal carotid artery, may explain the lower treatment effect seen in our study than in the SWIFT PRIME, ESCAPE, and EXTEND IA studies. The magnitude of benefit that we saw in our study was similar to that observed in MR CLEAN, with which our study shared many features.

Our sample size was not sufficient to permit robust assessments of the effects of thrombectomy in the prespecified subgroups. However, similar to the findings of previous trials,,$^{1,2,4}$ the positive treatment effect in our study appeared to be generally consistent across nearly all prespecified subgroups, including those defined according to NIHSS score, vessel-occlusion site, baseline ASPECTS score, and administration of intravenous alteplase. The benefits appeared to be less consistent in subgroups that were defined according to age and time window. Metaanalyses of data from multiple trials ${ }^{15}$ will be required to clarify the benefits of thrombectomy in such subgroups.

In our study, thrombectomy with the Solitaire 


\begin{tabular}{|c|c|c|c|c|}
\hline \multirow[t]{2}{*}{ Variable } & $\begin{array}{l}\text { Thrombectomy } \\
\text { (N=103) }\end{array}$ & $\begin{array}{l}\text { Control } \\
(N=103)\end{array}$ & $\begin{array}{l}\text { Between-Group Difference } \\
(95 \% \mathrm{Cl})\end{array}$ & $\begin{array}{l}\text { Risk Ratio } \\
(95 \% \mathrm{CI})\end{array}$ \\
\hline & \multicolumn{2}{|c|}{ no. (\%) } & & \\
\hline \multicolumn{5}{|l|}{ Safety variable } \\
\hline \multicolumn{5}{|l|}{ Death } \\
\hline At 90 days & $19(18.4)$ & $16(15.5)$ & $-2.9(-13.2$ to 7.3$)$ & $1.2(0.6$ to 2.2$) \dagger$ \\
\hline At $\leq 7$ days & $10(9.7)$ & $5(4.9)$ & $-4.8(-11.9$ to 2.2$)$ & $2.0(0.7$ to 5.6$)$ \\
\hline \multicolumn{5}{|l|}{ Intracranial hemorrhage } \\
\hline \multicolumn{5}{|l|}{ Symptomatict } \\
\hline SITS-MOST criteria & $2(1.9)$ & $2(1.9)$ & $0.0(-3.8$ to 3.8$)$ & $1.0(0.1$ to 7.0$)$ \\
\hline ECASS II criteria & $5(4.9)$ & $2(1.9)$ & $-2.9(-7.8$ to 2.0$)$ & $2.5(0.5$ to 12.6$)$ \\
\hline Asymptomatic $\mathbb{S}$ & $17(16.5)$ & $11(10.7)$ & $-5.8(-15.2$ to 3.5$)$ & $1.5(0.7$ to 3.1$)$ \\
\hline Subarachnoid hemorrhage & $5(4.9)$ & $2(1.9)$ & $-2.9(-7.8$ to 2.0$)$ & $2.5(0.5$ to 12.6$)$ \\
\hline \multicolumn{5}{|l|}{ Parenchymal hematoma } \\
\hline Any & $6(5.8)$ & $6(5.8)$ & & \\
\hline Type 1 & $3(2.9)$ & $4(3.9)$ & & \\
\hline Type 2 & $3(2.9)$ & $2(1.9)$ & & \\
\hline \multicolumn{5}{|l|}{ Other adjudicated serious adverse event } \\
\hline Neurologic worsening $\|$ & $16(15.5)$ & $13(12.6)$ & $-2.9(-12.4$ to 6.6$)$ & $1.2(0.6$ to 2.4$)$ \\
\hline Malignant cerebral edema*** & $11(10.7)$ & $10(9.7)$ & $-1.0(-9.2$ to 7.3$)$ & 1.1 (0.5 to 2.5$)$ \\
\hline Recurrent stroke & $4(3.9)$ & $3(2.9)$ & $-1.0(-5.9$ to 4.0$)$ & $1.3(0.3$ to 5.8$)$ \\
\hline Procedure-related complication $\dagger \hat{\dagger}$ & & NA & NA & NA \\
\hline Distal embolization in a different territory & $5(4.9)$ & & & \\
\hline Arterial dissection & $4(3.9)$ & & & \\
\hline Arterial perforation & $5(4.9)$ & & & \\
\hline Groin hematoma & $11(10.7)$ & & & \\
\hline Groin pseudoaneurysm & $1(1.0)$ & & & \\
\hline Vasospasm requiring treatment & $4(3.9)$ & & & \\
\hline
\end{tabular}

* Negative values for the between-group difference favor the control group. The risk ratio is for the thrombectomy group as compared with the control group. A complete list of adverse events is provided in Tables S6 and S7 in the Supplementary Appendix.

$\dagger \quad$ The adjusted risk ratio for death at 90 days was $1.1(95 \% \mathrm{Cl}, 0.8$ to 1.4$)$.

$\neq$ Symptomatic intracranial hemorrhage was defined as parenchymal hemorrhage type 2 on follow-up imaging and neurologic deterioration of at least 4 points on the NIHSS, according to the Safe Implementation of Thrombolysis in Stroke-Monitoring Study (SITS-MOST) criteria, or any symptomatic intracranial hemorrhage and neurologic worsening of at least 4 points on the NIHSS, according to the second European-Australasian Acute Stroke Study (ECASS II) criteria.

$\int$ Asymptomatic intracranial hemorrhage was defined as any parenchymal hematoma with no neurologic worsening, as adjudicated by local investigators.

I Parenchymal hematomas were graded according to the neuroimaging core laboratory classification.

\| Neurologic worsening was defined as an increase of at least 4 points on the NIHSS within 5 days after stroke onset that was not attributed to intracranial hemorrhage or malignant cerebral edema.

** Malignant cerebral edema was treated with decompressive hemicraniectomy in 3 patients in the thrombectomy group and in 6 patients in the control group.

tT All procedure-related complications were reported by the clinical events committee.

Vasospasm events were reported by local investigators and the angiography core laboratory.

stent retriever was found to be safe. Although there were no between-group differences in the rates of symptomatic intracranial hemorrhage (as defined according to SITS-MOST criteria) the observed early nonsignificant increase in mortality in the thrombectomy group, as compared with the control group, can be explained by multiple potential factors, including throm- 


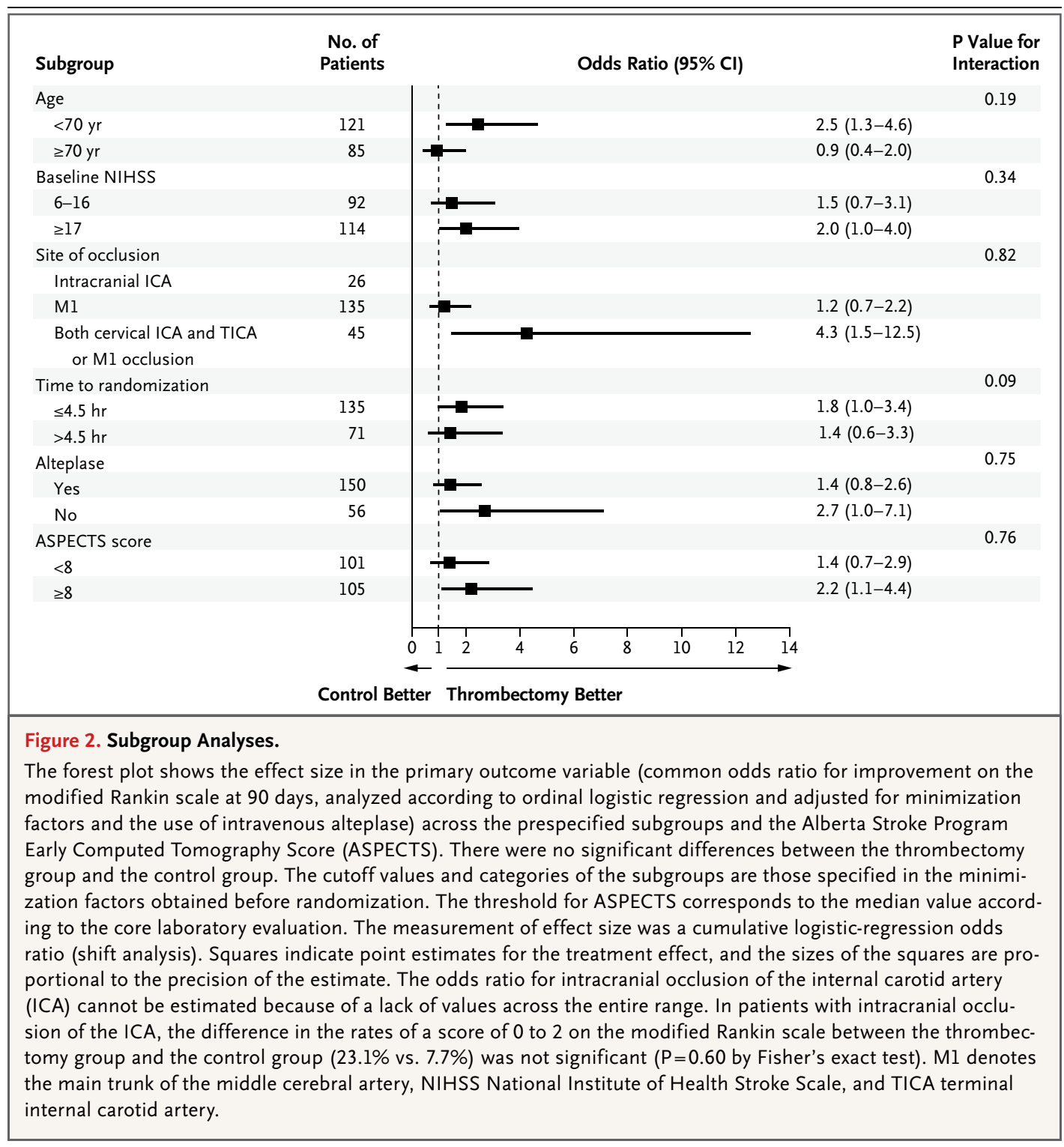

bectomy-associated adverse events. Nonetheless, at 90 days, the between-group difference in mortality was still not significant. Except for complications specific to thrombectomy, all of which were infrequent, major adverse event were evenly distributed between the two groups.

Our study has several limitations. Because of ethical considerations, the study was stopped before formal stopping boundaries were reached. In addition, the SONIIA registry captured only patients who were treated with reperfusion therapies and did not include all patients who were eligible for reperfusion therapies or who met the study criteria for eligibility.

In conclusion, our study contributes evidence to support the efficacy of neurovascular thrombectomy in patients with anterior circulation stroke who could be treated within 8 hours after the onset of symptoms.

Supported by Fundació Ictus Malaltia Vascular through an unrestricted grant from Covidien, by a grant from the Spanish Ministry of Health cofinanced by Fondo Europeo de Desarrollo Regional (Instituto de Salud Carlos III, Red Temática de Investigación Cooperativa Invictus, RD 12/0014/008), and a grant from the Generalitat de Catalunya (SGR 464/2014).

Disclosure forms provided by the authors are available with the full text of this article at NEJM.org. 


\section{APPENDIX}

The authors are as follows: Tudor G. Jovin, M.D., Angel Chamorro, M.D., Erik Cobo, Ph.D., María A. de Miquel, M.D., Carlos A. Molina, M.D., Alex Rovira, M.D., Luis San Román, M.D., Joaquín Serena, M.D., Sonia Abilleira, M.D., Ph.D., Marc Ribó, M.D., Mònica Millán, M.D., Xabier Urra, M.D., Pere Cardona, M.D., Elena López-Cancio, M.D., Alejandro Tomasello, M.D., Carlos Castaño, M.D., Jordi Blasco, M.D., Lucía Aja, M.D., Laura Dorado, M.D., Helena Quesada, M.D., Marta Rubiera, M.D., María Hernandez-Pérez, M.D., Mayank Goyal, M.D., Andrew M. Demchuk, M.D., Rüdiger von Kummer, M.D., Miquel Gallofré, M.D., and Antoni Dávalos, M.D.

The authors' affiliations are as follows: the Stroke Institute, Department of Neurology, University of Pittsburgh Medical Center, Pittsburgh (T.G.J.); the Stroke Unit (A.C., X.U.) and Department of Radiology (L.S.R., J.B.), Hospital Clínic, Department of Biostatistics, Universitat Politècnica de Catalunya (E.C.), Department of Radiology (M.A.M., L.A.) and Stroke Unit (P.C., H.Q.), Hospital de Bellvitge, L'Hospitalet de Llobregat, the Stroke Unit (C.A.M., M. Ribó, M. Ribiera) and Department of Radiology (A.R., A.T.), Hospital Vall d'Hebrón (J.S.), the Stroke Program, Agency for Health Quality and Assessment of Catalonia (S.A., M.G.), and the Department of Neuroscience and Trial Office Coordination, Hospital Germans Trias i Pujol, Universitat Autònoma de Barcelona (M.M., E.L.-C., C.C., L.D., M.H-P., A.D.) - all in Barcelona; the Stroke Unit, Hospital Josep Trueta, Ginona, Spain (J.S.); the Departments of Radiology and Clinical Neurosciences, Hotchkiss Brain Institute, Cumming School of Medicine, University of Calgary, Calgary, AB, Canada (A.M.D., M.G.); and the Department of Radiology, University of Dresden, Dresden, Germany (R.K.).

\section{REFERENCES}

1. Saver JL, Goyal M, Bonafe A, et al. Stent-retriever thrombectomy after intravenous t-PA vs. $\mathrm{t}-\mathrm{PA}$ alone in stroke. $\mathrm{N}$ Engl J Med 2015;372:2285-95.

2. Goyal M, Demchuk AM, Menon BK, et al. Randomized assessment of rapid endovascular treatment of ischemic stroke. N Engl J Med 2015;372:1019-30.

3. Campbell BC, Mitchell PJ, Kleinig TJ, et al. Endovascular therapy for ischemic stroke with perfusion-imaging selection. N Engl J Med 2015;372:1009-18.

4. Berkhemer OA, Fransen PS, Beumer

$\mathrm{D}$, et al. A randomized trial of intraarterial treatment for acute ischemic stroke. N Engl J Med 2015;372:11-20. [Erratum, N Engl J Med 2015;372:394.]

5. Goyal M, Almekhlafi M, Menon B, et al. Challenges of acute endovascular stroke trials. Stroke 2014;45:3116-22.

6. Abilleira S, Cardona $\mathrm{P}$, Ribó $\mathrm{M}$, et al. Outcomes of a contemporary cohort of 536 consecutive patients with acute ischemic stroke treated with endovascular therapy. Stroke 2014;45:1046-52.

7. Molina CA, Chamorro A, Rovira A, et al. REVASCAT: a randomized trial of revascularization with SOLITAIRE FR device vs. best medical therapy in the treatment of acute stroke due to anterior circulation large vessel occlusion presenting within eight-hours of symptom onset. Int J Stroke 2013 November 10 (Epub ahead of print).

8. Saver JL. Novel end point analytic techniques and interpreting shifts across the entire range of outcome scales in acute stroke trials. Stroke 2007;38:305562.

9. Kelly AG, Hoskins KD, Holloway RG. Early stroke mortality, patient preferences, and the withdrawal of care bias. Neurology 2012;79:941-4.

10. Wilson JT, Hareendran A, Grant M, et al. Improving the assessment of outcomes in stroke: use of a structured interview to assign grades on the modified Rankin Scale. Stroke 2002;33:2243-6.

11. Wahlgren N, Ahmed N, Dávalos A, et al. Thrombolysis with alteplase for acute ischaemic stroke in the Safe Implementation of Thrombolysis in Stroke-Monitor- ing Study (SITS-MOST): an observational study. Lancet 2007;369:275-82. [Erratum, Lancet 2007;369:826.]

12. Hacke W, Kaste M, Fieschi C, et al. Randomised double-blind placebo-controlled trial of thrombolytic therapy with intravenous alteplase in acute ischaemic stroke (ECASS II). Lancet 1998;352:124551.

13. Broderick JP, Palesch YY, Demchuk $\mathrm{AM}$, et al. Endovascular therapy after intravenous $t-P A$ versus $t-P A$ alone for stroke. N Engl J Med 2013;368:893-903. [Erratum, N Engl J Med 2013;368:1265.] 14. McTaggart RA, Jovin TG, Lansberg MG, et al. Alberta Stroke Program Early Computed Tomographic Scoring performance in a series of patients undergoing computed tomography and MRI: reader agreement, modality agreement, and outcome prediction. Stroke 2015;46:407-12. 15. Khatri P. The state of acute endovascular therapy: a report from the 12th TTST Conference. Stroke (in press). Copyright (c) 2015 Massachusetts Medical Society.

RECEIVE IMMEDIATE NOTIFICATION WHEN AN ARTICLE

IS PUBLISHED ONLINE FIRST

To be notified by e-mail when Journal articles are published Online First, sign up at NEJM.org. 
Reproduced with permission of the copyright owner. Further reproduction prohibited without permission. 